SUBJECT AREAS:

GASTRIC CANCER

DIAGNOSTIC MARKERS

Received

25 September 2013

Accepted

4 February 2014

Published

20 February 2014

Correspondence and requests for materials should be addressed to W.J.T. (twj8267@

sina.com) or Y.H.J.

(13945676987@

126.com)

* These authors contributed equally to this work.

\title{
Serum Helicobacter pylori NapA antibody as a potential biomarker for gastric cancer
}

\author{
Jingjing Liu' ${ }^{*}$, Huimin Liu'2*, Tingting Zhang ${ }^{1}$, Xiyun Ren ${ }^{1}$, Christina Nadolny ${ }^{3}$, Xiaoqun Dong ${ }^{3}$, \\ Lina Huang' ', Kexin Yuan', Wenjing Tian' \& Yunhe Jia ${ }^{4}$
}

\begin{abstract}
'Department of Epidemiology, College of Public Health, Harbin Medical University, Harbin, Heilongiiang Province, P. R. China, ${ }^{2}$ Department of neurology, The forth affiliated hospital, Harbin Medical University, Harbin 150001, Heilongjiang Province, P. R. China, ${ }^{3}$ Department of Biomedical and Pharmaceutical Sciences, College of Pharmacy, The University of Rhode Island, Pharmacy Building, 7 Greenhouse Road, Kingston, RI 02881, USA, 'Department of Colorectal Cancer Surgery, The third affiliated hospital, Harbin Medical University, Harbin 150081, Heilongiiang Province, P. R. China.
\end{abstract}

Helicobacter pylori (H. pylori) infection is strongly associated with gastric cancer. However, only a minority of infected individuals ever develop gastric cancer. This risk stratification may be in part due to differences among strains. The relationship between neutrophil-activating protein (NapA) and gastric cancer is unclear. The purpose of this study is to evaluate the significance of NapA as a biomarker in gastric cancer. We used enzyme linked immunosorbent assay (ELISA) to determine the status of $H$. pylori infection. Indirect ELISA method was used for detection of NapA antibody titer in the serum of $H$. pylori infected individuals. Unconditional logistic regressions were adopted to analyze the variables and determine the association of NapA and gastric cancer. The results of study indicated serum $H$. pylori NapA antibody level were associated with a reduced risk for development of gastric cancer. It may be used in conjugation with other indicators for gastric cancer detection.

\footnotetext{
$\longrightarrow$ astric cancer is one of the leading types of cancer and the second leading cause of cancer-related death worldwide, particularly in East Asian populations ${ }^{1-4}$. It has been calculated that the risk of gastric cancer in H. pylori-infected individuals is 3 to 6 fold higher than in those who are not infected ${ }^{5}$. H. pylori, a Gram-negative bacterium that colonizes the gastric mucosa of humans, has been recognized as the major etiological agent of chronic gastritis, peptic ulcer disease, and gastric cancer ${ }^{6,7}$. A number of trials have demonstrated the possibility of cancer prevention through $H$. pylori screening and eradication, particularly in high-risk populations (those with atrophic gastritis, intestinal metaplasia, dysplasia of the stomach, etc.). In a meta-analysis, the relative risk of gastric cancer following $H$. pylori eradication was calculated to be 0.65 overall $^{8}$. Therefore, its eradication seems a logical step in the prevention of gastric cancer. However, although more than $50 \%$ of the world population is infected with this bacterium, less than $2 \%$ develop gastric cancer ${ }^{2}$. In screening, the burden for patients as well as the ethics and the cost-effectiveness need to be taken into consideration. Hence, the strategy of widespread screening and eradication of $H$. pylori for gastric cancer seems inappropriate, and the screening of gastric cancer should be strictly limited to those with established indication of $H$. pylori infection. Currently, there is no sensitive biomarker for H. pylori related gastric cancer detection. Common makers such as CA19-9, fetoprotein antigen, pepsinogen I/II, and carcinoembryonic antigen (CEA) are prone to a high degree of false negatives $^{9,10}$. Therefore, novel markers are urgently required in order to detect $H$. pylori related gastric cancer. Moreover, it has been confirmed that $H$. pylori related gastric cancer is correlated with various virulence factors of H. pylori $i^{11,12}$.

H. pylori neutrophil-activating protein (HP-NapA), an important virulence factor that exists in all $H$. pylori strains, is associated with adhesion of the bacteria and inflammation occurrence ${ }^{13,14}$. In addition, NapA antibody was found more frequently in patients' sera of $H$. pylori infection. In the present study, we aim to evaluate the relationship between NapA and $H$. pylori related gastric cancer and discuss whether it can be used as a simple and reliable biomarker for the screening and diagnosis of gastric cancer patients.
} 
Table 1 | Demographic characteristics and $H$. pylori detection results of subjects

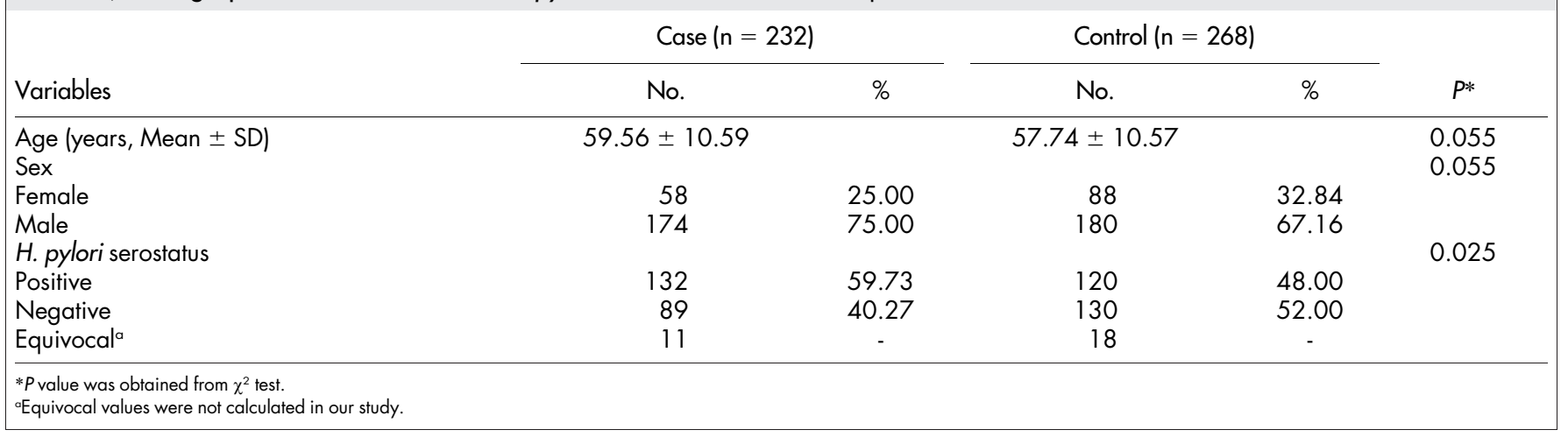

\section{Results}

Expression and purification of recombinant protein. The gene encoding for HP-NapA was obtained by PCR amplification using genomic DNA extracted from $H$. pylori clinical isolate template. A 435 bp DNA fragment, produced by PCR, was analyzed by agarose gel electrophoresis. Findings were consistent with expected results, including napA/pMD-18T detection and confirmation that the napA gene fragment was $435 \mathrm{bp}$. After ligating with expression vector pGEX-4T-1, the recombinant vector napA-pGEX-4T-1 was transformed into E.coli BL21DE3 cells. Nucleotide homology of napA from $H$. pylori clinical isolate strains compared with other standard H. pylori strains on the GenBank was $94 \%$ $98 \%$ using the BioEdit homology search tool.

The recombinant vector napA-pGEX-4T-1 was transformed into E.coli BL21DE3 cells to express fusion protein NapA, which was examined by sodium dodecyl sulfate polyacrylamide gel electrophoresis (SDS-PAGE). A pronounced band with an approximate molecular weight of $42 \mathrm{kDa}$ appeared in the supernatant of cell lysate after induction. This band did not appear in control cells, suggesting that the fusion protein can be successfully expressed in bacterial cells. Subsequently, the expressed fusion protein NapA was purified with a GST affinity column. The purified protein was analyzed by SDSPAGE, with a purity reaching more than 95\% (Supplementary Fig. S1).

Comparison of Helicobacter pylori infection results. H. pylori infection in serum samples of gastric cancer cases and controls were analyzed by an ELISA kit. The main demographic characteristics in study subjects and the detection results of $H$. pylori were shown in Table 1. There were no differences in sex and age between the cases and controls (age, $p=0.055$; sex, $p=0.055$ ). Among the 232 cases, $14(7.22 \%)$ were classified as stage I, 16 $(8.25 \%)$ as stage II, $143(73.71 \%)$ as stage III, and $21(10.82 \%)$ as stage IV, respectively. Only 9 of 232 patients (3.9\%) were gastric cardia cancer. The seroprevalence of $H$. pylori was $59.73 \%$ (132/ $221)$ in gastric cancer patients, compared with $48.00 \%(120 / 250)$ in control subjects. The seroprevalence of $H$. pylori in gastric cancer patients was significantly higher than the control group $(p=0.025)$.

Association between seropositivity of NapA antibody and GC. The NapA antibody titer was detected by indirect ELISA in $H$. pylori infected populations (132 gastric cancer patients and 120 control subjects). The results of NapA antibody levels and clinicopathologic factors of the $H$. pylori-infected individuals were shown in Table 2 . Among the $H$. pylori seropositivity subjects, NapA seropositivity was $12.12 \%$ (16 of 132) in gastric cancer patients and $1.67 \%(2$ of 120) in controls, respectively. A box plot showed the distribution of NapA antibody levels of cases and controls infected by H. pylori (Supplementary Fig. S2). To examine the factors affecting the relationship between gastric cancer risk and NapA antibody, ORs (odds ratios) and 95\% CIs (confidence intervals) were estimated in the $252 \mathrm{H}$. pylori positive cases and control subjects by both unconditional univariate and multivariate logistic regression analyses. As shown in Table 3, smoking (OR $=2.55$; 95\% CI, $1.50-$ $4.35 ; p<0.01)$ and alcohol $(\mathrm{OR}=2.64$; 95\% CI, $1.56-4.47 ; p<$ $0.01)$ were associated with an increased risk of gastric cancer in patients compared with controls based upon unconditional univariate logistic regression analysis. The associations between GC and seropositivity of NapA antibody were significant with OR (95\% CI) of 9.21 (1.96-43.14) while controlling for age, sex, smoking and alcohol drinking $(P<0.01)$.

Screening utility of serum NapA antibody to GC. The receiver operating characteristic (ROC) curve was plotted to evaluate the screening utility and identify a cut-off point of serum NapA antibody result, as its value was considered as an independent predictor of GC among the $H$. pylori-positive population. According to the ROC curve, the optimal cutoff value for GC was 0.146 , providing a sensitivity of $86.36 \%$ and a specificity of $35.00 \%$. When the NapA cutoff value (OD) was 0.201 , the sensitivity of serum NapA antibody was $97.73 \%$ and specificity was $10.00 \%$. With the cutoff value set at 0.065 , the corresponding sensitivity and specificity were $15.15 \%$ and $95.00 \%$, respectively (Table 4 ). The area under the receiver operating characteristic curve (AUC) for NapA was 0.63 (95\%CI, 0.56-0.70; Supplementary Fig. S3).

\section{Discussion}

Helicobacter pylori infection has been established as a major risk factor for gastric cancer, and the World Health Organization classified H. pylori infection as a definite (class I) carcinogen in $1994^{2,15}$. Previous studies have confirmed that the incidence of gastric cancer could be decreased by eradication of $H$. pylori infection ${ }^{16}$. The prevention of gastric cancer by $H$. pylori eradication in healthy subjects is conducted around the world. For example, a large-scale intervention study of $H$. pylori eradication on gastric cancer prevention had been conducted in China ${ }^{17}$. The study included 1,630 patients with $H$. pylori infection and followed up for 7.5 years. A prophylaxis effect of H. pylori eradication for gastric cancer was observed ${ }^{16}$. In addition, while screening by endoscopy or photofluorography is currently employed in high-incidence populations, testing of serum biomarkers for $H$. pylori will provide a more convenient, cost-effective and noninvasive screening method that can be practiced worldwide. Many investigators and clinicians have therefore sought to find accurate and sensitive noninvasive serum markers against $H$. pylori related gastric cancer, but this remedy has yet to be found. Novel serum markers are thus urgently required to identify ways to screen H. pylori infection for eradication and prevent gastric cancer formation.

In this paper, the seropositivity of $H$. pylori in 232 cases with gastric cancer was significantly higher compared with 268 controls 
Table 2 | NapA antibodies levels and clinicopathological factors of the $H$. pylori-infected individuals

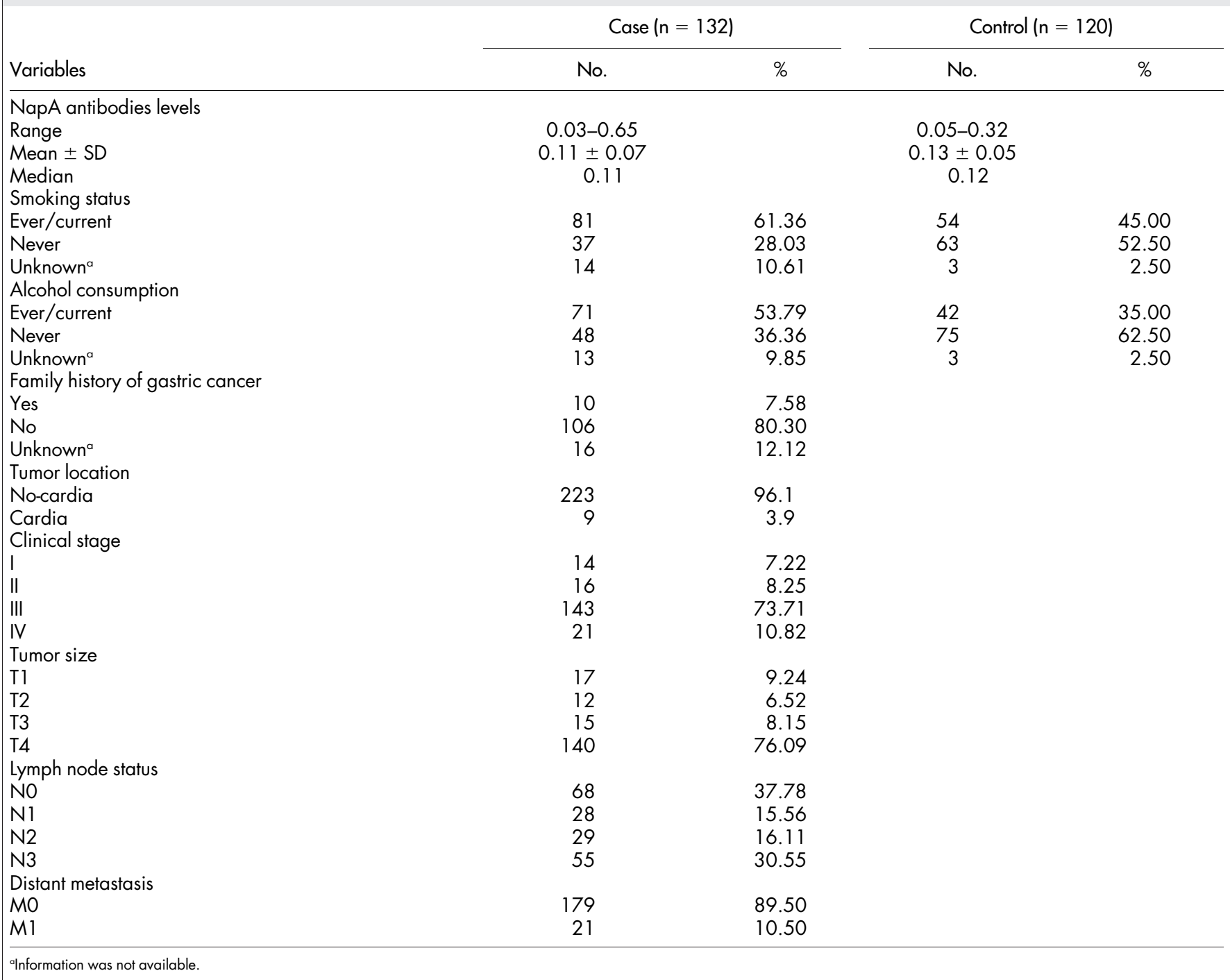

determined using an ELISA. This confirmed that $H$. pylori infection increased the risk of gastric cancer, which was in line with previous studies ${ }^{2,18}$. It has been reported that prevalence of $H$. pylori was previously high in China but has been declining over recent decades, varying by geographic locations. For the control group, seropositivity of $H$. pylori was $48.00 \%$, which was lower than that in Muping (50.95\%), but higher than that in Yanqing $(41.35 \%)^{19}$. For the GC patients, seropositivity of $H$. pylori was $59.73 \%$, which was close to that in Taiwan $(60.9 \%)^{20}$ and German $(66.1 \%)^{21}$, but higher than that in Greece $(34.9 \%)^{22}$, and lower than that in Japan
$(82.0 \%)^{23}$. However, the prevalence of $H$. pylori infection might be underestimated due to disease-related clearance of $H$. pylori infection in the past or the spontaneous disappearance of the bacterium from the gastric mucosa during the progression of gastric atrophy precancerous lesions ${ }^{24}$. It should be noticed that seropositivity of $H$. pylori in a hospital-based case-control study cannot reflect the accurate $H$. pylori prevalence in the population. Although it can reflect the $H$. pylori prevalence to some extent, we should be careful to compare the results to those from populationbased studies.

Table 3 | Univariate and Multivariate models to detect potential risk factors for gastric cancer in subjects infected by $\mathrm{H}$. pylori

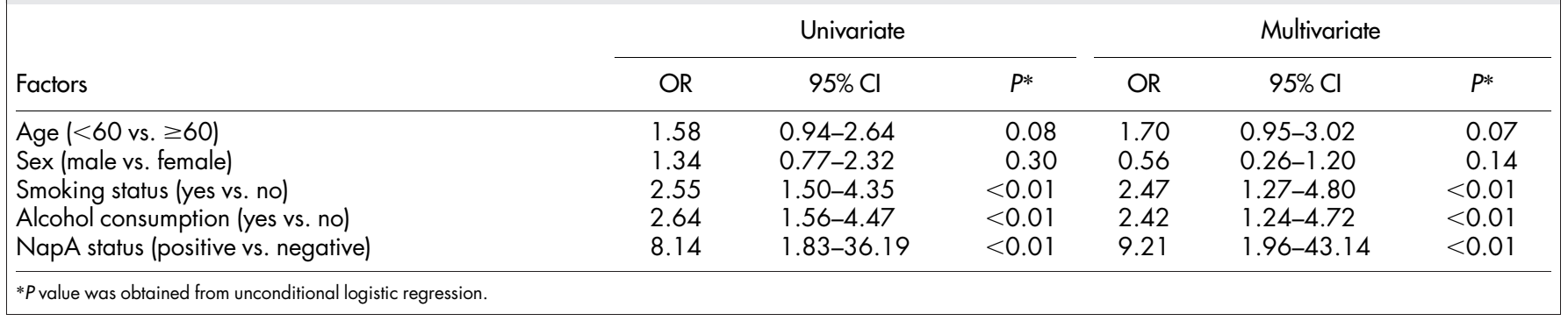




\begin{tabular}{|c|c|c|c|}
\hline Percentile $^{1}$ & $\begin{array}{l}\text { NapA critical } \\
\text { value (OD) }\end{array}$ & $\begin{array}{l}\text { Sensitivity }(\%) \text { case test }{ }^{+} / \text {(case } \\
\text { test }^{+}+\text {case test }^{-} \text {) }\end{array}$ & $\begin{array}{c}\text { Specificity (\%) control test } \\
\text { test }^{+} / \text {( control } \\
\text { (control test }\end{array}$ \\
\hline $90 \%$ & 0.201 & 97.73 & 10.00 \\
\hline $75 \%$ & 0.159 & 89.39 & 24.17 \\
\hline $50 \%$ & 0.121 & 62.88 & 49.17 \\
\hline $5 \%$ & 0.065 & 15.15 & 95.00 \\
\hline
\end{tabular}

'Percentiles of serum NapA antibody levels in controls.

Identification of the optimal cut-off point in the different parameters was performed according to the maximum Youden's index (sensitivity + specificity -1 ).

What's more, accumulating evidence has indicated that $H$. pylori infection could increase the risk of gastric non-cardia cancer but was not or even inversely associated with the risk of gastric cardia can$\operatorname{cer}^{25,26}$. Our study included 9 (3.9\%) gastric cardia cancer cases; however, their involvement did not affect the overall results and conclusion.

In the present study, we explored the significance and the processes of NapA, a virulence factor of $H$. pylori, as a serum biomarker in gastric cancer.

We cloned and expressed HP-NapA, which was then used as an antigen to detect HP-NapA-specific antibodies in serum samples. The levels of HP-NapA antibodies were detected in $132 \mathrm{H}$. pyloriinfected gastric cancer patients and 120 control subjects. As a result, the unadjusted seropositivity of HP-NapA in patients was significantly higher than in controls. Moreover, after adjustment for age, gender, alcohol consumption and smoking history, seropositivity remained significant. Although a significant difference was found for the seropositivity of HP-NapA antibody in the two groups, the median of HP-NapA antibody levels was only slightly lower in patients than the controls ( 0.11 vs 0.12$)$, which indicates high antibody titer HP-NapA may protect from the development of gastric cancer.

An AUC value between 0.5-0.7 is considered as lower accuracy, $0.7-0.9$ with certain accuracy, and greater than 0.9 with high accuracy. In our study, the AUC was 0.63. These suggests that although NapA can be used as a serum biomarker for the screening of $H$. pylori infection, NapA does not seem to be an independent biomarker for detecting high risk populations from $H$. pylori-infected individuals who are susceptible to gastric cancer development.

On the other hand, Kountouras J et al. found that NapA was potentially correlated with gastric carcinogenesis, but failed to establish any significance between the level of serum NapA antibody and gastric cancer ${ }^{27,28}$. Recently, Choli-Papadopoulou et al. reported the development of new drugs targeting H. pylori $\mathrm{NapA}^{27,28}$. Our findings were in line with a study conducted by Min Long et al. who found significant correlation between serum NapA antibodies level in $H$. pylori-infected patients and healthy controls ${ }^{29,30}$. They showed the seropositivity for HP-NapA specific antibodies in gastric cancer patients was higher than that in chronic gastritis patients and healthy controls. However, multivariate analysis was not performed completely in their study, and thus it was unknown if confounding factors, such as gender and smoking, contributed to the differences observed. Moreover, the sample size was small $(\mathrm{n}=188$, including cases and controls) and the seropositivity for antibodies against HP-NapA in gastric cancer was remarkably higher (97.7\%).

NapA as an important virulence factor of $H$. pylori increases the adhesion of neutrophils to endothelial cells. The significant relationship between NapA antibody level and gastric cancer risk suggests that NapA forebodes a substantial down-regulation effect in the development of gastric cancer and HP-NapA low antibody titer predicts susceptibility to gastric cancer. However, the low AUC indicates that NapA cannot be used as an independent screening and diagnosis criterion of gastric cancer. It may be used in conjunction with other assays for $H$. pylori screening and early detection for gastric cancer. Other virulence factors of $H$. pylori such as CagA or VacA were not evaluated at the same time with NapA as parallel comparisons were a limitation to this study. More prospective studies should be carried out with larger sample sizes to identify the ideal, inexpensive and readily available biological markers for gastric cancer. Further investigations are needed to evaluate the feasibility of NapA antibody as a biomarker in population-based screening for gastric cancer.

\section{Methods}

Subjects. A case-control study was designed to evaluate the significance of NapA as a biomarker for gastric cancer. This study included 232 gastric cancer patients ( 58 women and 174 men, ages 33-83, Table 1) who were newly diagnosed by the Third Affiliated Hospital of Harbin Medical University between March-July, 2010. The control group consisted of 184 healthy physical examination individuals and 84 cancer-free neurological patients, respectively ( 88 women and 180 men, ages 35-82, Table 1). Moreover, all participants completed a face-to-face questionnaire regarding sociodemographic characteristics, health status, lifestyle factors et al. Questionnaires were recorded by two investigators independently. In addition, informed consent was obtained from all participants and all procedures were carried out in accordance with the guidelines by the Human Research and Ethics Committee of Harbin Medical University. For standardization of the study all blood samples were collected by the same venipuncture staff, each in $5 \mathrm{~mL}$ vacutainer tubes containing EDTA as an anticoagulant. All blood samples were centrifuged and stored at $-80^{\circ} \mathrm{C}$ until assayed.

Cloning and purification of HP-NapA. A clinical strain of H. pylori, isolated from a gastric cancer patient at the Second Affiliated Hospital of Harbin Medical University, was used and provisionally named HLJ016 by our group. Genomic DNA of HLJ016 was extracted using a DNA extraction kit (QIAGEN, USA) and stored at $-80^{\circ} \mathrm{C}$. The extracted genomic DNA was then used as a template for amplification of the napA coding region using a Taq DNA polymerase PCR kit (Takara, Biotechnology of DALIAN). Two primer sequences corresponding to the $5^{\prime}$ and $3^{\prime}$ ends of the coding gene $^{30}$, including EcoR I and Xho I restriction sites. The PCR product was cloned into the cloning vector pMD18-T and then transformed into E.coli strain DH5 $\alpha$. The positive clones were screened by PCR and restriction enzyme digestion. DNA sequence of the amplified napA gene was assayed after sequenced by using the dideoxy chain termination method (biotechnology firm, BGI) then cloned into the expression vector $\mathrm{pGEX}-4 \mathrm{~T}-1$. The recombinant plasmid napA/pGEX-4T-1 was then transformed into E.coli BL21DE3 cells, confirmed by PCR and the same restriction enzyme digestion, and the target DNA sequence of napA gene was assayed again using the same method. The recombinant strain pGEX-4T-1-napA-BL21DE3 was cultured in LB medium with $100 \mu \mathrm{g} / \mathrm{mL}$ ampicillin, and then induced by IPTG with a final concentration of $0.5 \mathrm{mmol} / \mathrm{L}$ at $25^{\circ} \mathrm{C}$. E.coli cells were harvested after 6 hours and lysed via ultrasonication. The suspension was collected and examined by $15 \%$ sodium dodecyl sulfate polyacrylamide gel electrophoresis (SDS-PAGE). The recombinant protein was purified with GST affinity resin (GENMED SCIENTIFICS INC, USA).

Serologic tests of the $\boldsymbol{H}$. pylori by ELISA. A serologic test for H. pylori IgG antibodies was run in duplicate using an enzyme immunoassay kit (IBL, German). Sensitivity and specificity of the kit, given by the manufacturer, were all greater than $95 \%$. According to the manufacturer's instruction, 101-fold diluted serum was measured, with $<8$ units/ml considered as negative, $8-12$ units $/ \mathrm{ml}$ as equivocal, and $>12$ units/ $\mathrm{ml}$ as positive.

Detection of antibodies against HP-NapA in serum with ELISA. An ELISA was performed according to previously published documents using prepared and purified recombinant HP-NapA ${ }^{31-34}$. Briefly, the purified NapA protein was diluted to $4 \mu \mathrm{g} /$ $\mathrm{ml}$ and incubated overnight at $4{ }^{\circ} \mathrm{C}$. Plates were then washed five times with a PBS 
buffer and blocked with 7\% goat serum (AR0009, BOSTER) and incubated in a moist chamber for $2 \mathrm{~h}$ at $37^{\circ} \mathrm{C}$. Plates were again washed five times, each time for 3 minutes. $300 \mu \mathrm{L} /$ well with buffer, serum samples (1:50) from patients or control individuals, were then added to the microtiter wells, and the plates were incubated at $37^{\circ} \mathrm{C}$ for 60 min. After washing with PBS, $100 \mu \mathrm{L}$ Peroxidase-Conjugated Goat Anti-Human IgG (ZB-2304, ZSGB-BIO), diluted $1: 40,000$ in buffer, was added to each well and incubated for $60 \mathrm{~min}$ at $37^{\circ} \mathrm{C}$. The plates were washed with PBS and TMB substrate $(100 \mu \mathrm{L})$ was added to the wells and incubated at room temperature for $15 \mathrm{~min}$, free of light. The reaction was terminated by adding $50 \mu \mathrm{L}$ of stop solution. The absorbance of each well was read at $450 \mathrm{~nm}$ using a microplate reader (BioTek Synergy2, USA). Samples with absorbance OD $\leq$ mean - 2 standard deviations (SDs) of controls who infected by $H$. pylori were considered positive.

Statistical analysis. SPSS version 18.0 was used for all statistical analysis. Distributions of variables and rates of $\mathrm{H}$. pylori and NapA antibody were computed. The $p$ value $<0.05$ was used to determine the difference of rates among the groups of gastric cancer and controls. Unconditional univariate and multivariate logistic regression analyses were used to estimate $\mathrm{OR}$ and $95 \% \mathrm{CI}$ for the relation in gastric cancer and NapA antibody considering the potential confounders in H. pylori infected individuals. Means, medians, and full ranges of $H$. pylori and NapA antibody levels of cases and controls were computed and compared with parametric test. ELISA data were transformed by a logarithm function firstly, and then tested by the K-S Test. Cut-off point was determined according to the mean -2 standard deviations (SDs) of controls who infected by $H$. pylori. Additionally, sensitivity, specificity and area under the receiver operating characteristic curve (AUC) with $95 \%$ confidence intervals (CI) were computed to evaluate the value of serum NapA antibody levels for screening high risk population prone to gastric cancer.

1. Hamilton, J. P. \& Meltzer, S. J. A review of the genomics of gastric cancer. Clin. Gastroenterol. Hepatol. 4, 416-425 (2006).

2. Conteduca, V. et al. H. pylori infection and gastric cancer: state of the art (review). Int. J. Oncol. 42, 5-18 (2013).

3. Yang, X. X. et al. Association of genetic polymorphisms at 1q22 but not 10q23 with gastric cancer in a southern Chinese population. Asian Pac. J. Cancer Prev. 13, 2519-2522 (2012).

4. Thiel, A. \& Ristimaki, A. Gastric cancer: basic aspects. Helicobacter. 17 Suppl 1, 26-29 (2012).

5. Kim, S. S., Ruiz, V. E., Carroll, J. D. \& Moss, S. F. Helicobacter pylori in the pathogenesis of gastric cancer and gastric lymphoma. Cancer Lett. 305, 228-238 (2011).

6. Dundon, W. G. et al. The neutrophil-activating protein of Helicobacter pylori. Int. J. Med. Microbiol. 291, 545-550 (2002).

7. Lamb, A. \& Chen, L. F. Role of the Helicobacter pylori-induced inflammatory response in the development of gastric cancer. J. Cell. Biochem. 114, 491-497 (2013).

8. Fuccio, L. et al. Meta-analysis: can Helicobacter pylori eradication treatment reduce the risk for gastric cancer? Ann. Intern. Med. 151, 121-128 (2009).

9. Chong, P. K. et al. Upregulation of plasma C9 protein in gastric cancer patients. Proteomics. 10, 3210-3221 (2010).

10. Lam, K. W. \& Lo, S. C. Discovery of diagnostic serum biomarkers of gastric cancer using proteomics. Proteomics Clin Appl. 2, 219-228 (2008).

11. Wen, S. \& Moss, S. F. Helicobacter pylori virulence factors in gastric carcinogenesis. Cancer lett. 282, 1-8 (2009).

12. Abdullah, S. M. et al. Infection with Helicobacter pylori strains carrying babA2 and cagA is associated with an increased risk of peptic ulcer disease development in Iraq. Arab J Gastroenterol. 13, 166-169 (2012).

13. D'Elios, M. M., Amedei, A., Cappon, A., Del Prete, G. \& de Bernard, M. The neutrophil-activating protein of Helicobacter pylori (HP-NAP) as an immune modulating agent. FEMS Immunol. Med. Microbiol. 50, 157-164 (2007).

14. Wang, C. A., Liu, Y. C., Du, S. Y., Lin, C. W. \& Fu, H. W. Helicobacter pylorineutrophil-activating protein promotes myeloperoxidase release from human neutrophils. Biochem. Biophys. Res. Commun. 377, 52-56 (2008)

15. Pounder, R. E. \& Ng, D. The prevalence of Helicobacter pylori infection in different countries. Aliment. Pharrnacol. Ther. 9, 33-39 (1995).

16. Asaka, M., Kato, M. \& Graham, D. Y. Prevention of Gastric Cancer by Helicobacter pylori eradication. Intern. Med. 49, 633-636 (2010).

17. Wong, B. C. et al. Helicobacter pylori eradication to prevent gastric cancer in a high-risk region of China: a randomized controlled trial. JAMA. 291, 187-194 (2004)

18. Prinz, C., Schwendy, S. \& Voland, P. Helicobacter pylori and gastric cancer: shifting the global burden. World J. Gastroenterol. 12, 5458-5464 (2006).
19. Zhang, D. H. et al. Recent changes in the prevalence of Helicobacter pylori infection among children and adults in high- or low-incidence regions of gastric cancer in China. Chin. Med. J. 122, 1759-1763 (2009).

20. Lee, W. J. et al. Clinicopathologic characteristics of Helicobacter pyloric seropositive gastric adenocarcinomas. J. Clin. Gastroenterol. 21, 203-207 (1995).

21. Gao, L. et al. Helicobacter pylori infection and gastric cancer risk: evaluation of 15 H. pylori proteins determined by novel multiplex serology. Cancer Res. 69, 6164-6170 (2009).

22. Syrios, J. et al. Survival in patients with stage IV noncardia gastric cancer-the influence of DNA ploidy and Helicobacter pylori infection. BMC cancer. 12, 264 (2012).

23. Barreto-Zuñiga, R. et al. Significance of Helicobacter pylori infection as a risk factor in gastric cancer: serological and histological studies. J. Gastroenterol. 32, 289-294 (1997).

24. Gonzalez, C. A. et al. Helicobacter pylori infection assessed by ELISA and by immunoblot and noncardia gastric cancer risk in a prospective study: the EurgastEPIC project. Ann. Oncol. 23, 1320-1324 (2012).

25. Helicobacter and Cancer Collaborative Group. Gastric cancer and Helicobacter pylori: a combined analysis of 12 case control studies nested within prospective cohorts. Gut. 49, 347-353 (2001).

26. Kamangar, F. et al. Opposing risks of gastric cardia and noncardia gastric adenocarcinomas associated with Helicobacter pylori seropositivity. J. Natl. Cancer Inst. 98, 1445-1452 (2006).

27. Kountouras, J. et al. Potential implications of Helicobacter pylori-related neutrophil-activating protein. World J. Gastroenterol. 18, 489-490 (2012).

28. Choli-Papadopoulou, T., Kottakis, F., Papadopoulos, G. \& Pendas, S. Helicobacter pylori neutrophil activating protein as target for new drugs against $H$. pylori inflammation. World J. Gastroenterol. 17, 2585-2591 (2011).

29. Long, M., Luo, J., Li, Y., Zeng, F. Y. \& Li, M. Detection and evaluation of antibodies against neutrophil-activating protein of Helicobacter pylori in patients with gastric cancer. World J. Gastroenterol. 15, 2381-2388 (2009).

30. Luo, J. et al. Clone, expression and immunoreaction of napA gene of Helicobacter pylori. Chin J Cell Mol Immunol. 23, 395-398 (2007).

31. Ohkusu, K. Cost-effective and rapid presumptive identification of gram-negative bacilli in routine urine, pus, and stool cultures: evaluation of the use of CHROMagar orientation medium in conjunction with simple biochemical tests. J. Clin. Microbiol. 38, 4586-4592 (2000).

32. Xiang, Z. et al. Detection in an enzyme immunoassay of an immune response to a recombinant fragment of the 128 kilodalton protein $(\mathrm{CagA})$ of Helicobacter pylori. Eur. J. Clin. Microbiol. Infect. Dis. 12, 739-745 (1993).

33. Kashyap, R. S. et al. Diagnosis of tuberculosis in an Indian population by an indirect ELISA protocol based on detection of Antigen 85 complex: a prospective cohort study. BMC Infect. Dis. 7, 74 (2007).

34. Tang, J. W. et al. Evaluation of Epstein-Barr virus antigen-based immunoassays for serological diagnosis of nasopharyngeal carcinoma. J. Clin. Virol. 40, 284-288 (2007).

\section{Acknowledgments}

This study was supported by National Natural Science Foundation of China (2009-2011 Grant No. 30800939).

\section{Author contributions}

W.J.T. and Y.H.J. designed the experiments. J.J.L., H.M.L. and T.T.Z. performed the experiments, analyzed the data, prepared the figures and tables and wrote the manuscript. The experiments were supported by C.N., X.Q.D., X.Y.R., L.N.H. and K.X.Y. J.J.L. and H.M.L. contributed equally to this paper. All the authors had read the manuscript, agreed that it was ready for publication and accepted responsibility for its contents.

\section{Additional information}

Supplementary information accompanies this paper at http://www.nature.com/ scientificreports

Competing financial interests: The authors declare no competing financial interests.

How to cite this article: Liu, J.J. et al. Serum Helicobacter pylori NapA antibody as a potential biomarker for gastric cancer. Sci. Rep. 4, 4143; DOI:10.1038/srep04143 (2014).

(1) (5) $\Theta$ This work is licensed under a Creative Commons AttributionBY NC ND NonCommercial-NoDerivs 3.0 Unported license. To view a copy of this license, visit http://creativecommons.org/licenses/by-nc-nd/3.0 